Ann. A beille, $\mathrm{Ig}_{4} \mathrm{7}(3), \mathrm{r} 8 \mathrm{r}-\mathrm{I} 85$.

\title{
DÉTERMINATION DE LA TENEUR EN CIRE MICROCRISTALLINE DES MÉLANGES DE CIRE D'ABEILLE ET DE CIRES MICROCRISTALLINES
}

\author{
Ph. VERGERON \\ Station de Recherches sur l'Abeille et les Insectes sociann, \\ Bures-sur-Yvette (Seine-et-Oise).
}

SOMMAIRE,

Les méthodes utilisées actuellement pour l'analyse des mélanges de cire d'abeille et de cires microcristallines sont compliquées et assez peu satisfaisantes. Une nouvelle méthode est proposét Elle consiste à établir le rapport hydrocarbures/alcools gras du mélange à étudier. Le pourcentage des microcristallines est alors déterminé en se référant à une courbe théorique vérifiée expérimen. talement. Les hydrocarbures et les alcools gras sont isolés par la méthode de A. Les's (I9I3).

\section{INTRODUCTION}

A l'heure actuelle, la cire d'abeille est parfois remplacée par des succédanés moins cô̂teux obtenus au cours des différentes opérations de raffinage des huiles lubrifiantes de pétrole. Des mélanges de cire d'abeille et de cire de pétrole ont été couranment mis en vente dans le commerce, parfois du reste sous des appellations ne faisant état que du premier composant. C'est pourquoi nous avons essayé, au cours de ces derniers mois, de mettre au point une méthode d'analyse simple et rapide. assurant un contrôle facile des produits considérés et donnant des résultats relativement précis. Ceci était d'autant plus nécessaire que les méthodes utilisées jusqu'alors étaient longues, peu précises et souvent infidèles (en particulier la détermination de l'indice d'hydroxyle). Reprenant des techniques analytiques d'Alexandre I.EYS une méthode plus convenable a été mise au point.

\section{Io Composition des microcristallines.}

L,es pétroles sont relativement riches en produits paraffineux. Ceux du MoyenOrient du type Aramco, courants en France il y a quelques années, en contiennent 
bruts 3,5 p. cent (M. CHAUVIX, I955). Ces substances paraffineuses donnent naissance à divers produits commerciaux connus sous les noms de paraffines, microcristallines et pétrolatum. Les méthodes classiques de " presse " et de "ressuage " donnent des cires paraffineuses raides et cassantes. Très employées ces dernières années par les apiculteurs, les cires microctistallines sont isolées au cours de la fabrication des huiles lubrifiantes raffinées et de la purification de certains produits résiduaires voisins des asphaltes (M. Chauvin, I955). Leur origine étant variable, la composition de ces cires 1'est également. Les spécialistes des industries pétrolières n'essaient guère d'obtenir des composés chimiquement définis mais des fractions surtout caractérisées par des tests physiques et industriels. I'industrie américaine définit les microcristallines non par des dosages chimiques mais seulement à l'aide de tests physiques (KANE, I960).

Ces cires microcristallines sont composées d'hydrocarbures à l'état presrue pur. Ce sont des paraffines ou des isoparaffines, parfois même des cycloparaffines conportant généralement de 23 à 28 atomes de carbone. Ies chaînes, linéaires ou non, de 18 à $2 \mathrm{I}$ atomes de carbone d'une part et de 30 à 43 d'autre part ont parfois été décelées (Fir.IS, I945). Ce sont les rapports liant ces différents composés qui sont responsables des propriétés relativement variées des produits ('TuRNIik et coll., I955 ; MAZEE, I949). A côté des composants principaux (hydrocarbures), se trouvent des impuretés qui peuvent cependant atteindre des proportions relativement importantes. I,es cérésines contiendraient des naphtènes (F,I,Is, I945). I)e plus, les cires microcristallines étant obtenues en grande partie au cours du raffinage des huiles, un contenu huileux subsiste avec les hydrocarbures. Peu important pour les paraffines (nous avons vérifié qu'il était toujours voisin de $\mathrm{I}$ p. cent comme la plupart des auteurs l'indiquent) il existe en proportions plus fortes dans les microcristallines. Cependant, l'industrie tente actuellement de limiter ce phénomène qui correspond pour elle à une perte d'huile lubrifiante qui est un produit cher. Alors qu'il y a quelques années, le contenu huileux pouvait atteindre plus de 30 p. cent (L,TIECKE et IVANovszky 1,1958 ), il semble qu'il se limite maintenant à des taux inférieurs.

Aucun autre composant n'est généralement indiqué. I) très faibles quantités d'esters ont été rarement décelées. Il semble cependant qu'il ne s'agisse que d'impuretés accidentelles dues à certains traitements particuliers (GRUsw et STEVENs, Ig6o).

Le commerce offre, à l'heure actuelle, des types de cires microcristallines variées répondant à des besoins divers. I,industrie papetière consomme de grandes quantités de dérivés cireux du pétrole ainsi que l'industrie des cosmétiques et du petit matériel électrique. L'apiculture n'est qu'un débouché mineur pour les cires microcristallines. Elle n'en utilise que certaines qualités possédant les propriétés indispensables à la fabrication de la cire gaufrée. I es plus couramment utilisées sont:

les cires microcristallines laminées blanches et jaunes

les cires Micro A 75 et M 85

la cire microcristalline $n^{0} 5$ à la cérésine

la cire Warco I80 raffinée

Tous ces produits ont été analysés au laboratoire. Nous avons étudié comparativement des paraffines susceptibles d'être également utilisées :

paraffines raffinées $50 / 5^{2}$ et $58 / 60$

paraffines " écaille " $48 / 56$ et $56 / 60$ 
$2^{\circ}$ Méthodes de dosage actuellemeut utilisées pour le contrôle de la cire d'abeille.

- Méthode classique de dosage :

L'insaponifiable est extrait de l'échantillon de cire au Kumagawa. Sur cet insaponifiable recueilli, on détermine l'indice d'hydroxyle selon les indications de DFIAHY et PEIGNOT (I948) (M.U.I.). L'indice d'hydroxyle est caractéristique des alcools non estérifiés de la cire d'abeille. Or, la présence de ces substances dépend de la quantité d'acides gras dans l'échantillon, quantité qui elle-même est assez. variable (l'étendue de cette variation atteint $20 \mathrm{p}$. cent de la valeur moyenne).

Il est difficile de vérifier que l'extraction au Kumagawa est bien totale. De plus, l'utilisation de l'insaponifiable extrait est assez peu commode. La technique de détermination de l'indice d'hydroxyle est compliquée et de plus donne des résultats peu fidèles sur la cire d'abeille. Enfin, la variation du taux d'alcools non estérifiés rend difficile une conclusion nette et précise.

- Autres méthodes :

De nombreuses autres méthodes peuvent être proposées. Celle des filtrations à température fixe de GASCARD et I)AMOY serait très valable, mais elle est longue et délicate. L'indice de saponification a servi à établir d'autres méthodes. Celle de PARKER est très subjective et ne permet de détecter que la présence ou l'absence de produit d'addition. Celle de SPENGI,ER et WEBER a l'avantage de considérer la quantité totale de paraffine mais cette quantité est elle-même rapportée à l'indice de saponification ou à l'indice d'ester qui sont très variables (Brsson et coll., I940). Enfin, ces derniers temps, White et coll. (I960) en Amérique et Fuchs et JoxG (I954) en Allemagne ont utilisé la méthode de chromatographie sur colonne. Mais les hydrocarbures isolés ne peuvent être pris en considération seuls comme le fait remarquer WhITE. De plus, la mesure du point de fusion des hydrocarbures préconisée par cet auteur est de toute façon influencée par la variation du taux de cette famille de corps dans les produits considérés et demande beaucoup trop de soins pour la précision qu’elle apporte.

\section{MÉTHODE PROPOSÉE}

I. Défunition du rapport $\stackrel{\mathrm{h}}{\mathrm{a}}$ :

Les hydrocarbures $(h)$ constituent les seules substances communes aux compositions de la cire d'abeille et des cires microcristallines; il est donc intéressant de les considérer. Non moins intéressants sont, d'après nous, les alcools gras $(a)$ qui représentent un constituant constant et, dans le cas présent, spécifique de la cire d'abeille.

En effet, le pourcentage d'alcools gras dans celle-ci est beaucoup plus constant. Nous sommes arrivés à des chiffres toujours compris entre 38 et 39,5 , valeurs retrouvées à peu près partout dans les travaux antérieurs. Donc ce taux d'alcools gras de la cire d'abeille semble à peu près fixe.

Nous avons essayé au laboratoire de détecter la présence possible d'alcools gras dans les cires microcristallines. Sur tous les échantillons étudiés, aucun produit ne s'est comporté comme tel. Seul le $n^{\circ} 5$ à la cérésine a donné naissance à une 
très faible pellicule au moment de l'isolement des alcools. Dans les autres cas, nous avons toujours obtenu un résultat négatif. De plus, aucun travail antérieur à notre connaissance ne parle de tels composants. Nous sommes donc pratiquement certain que les alcools gras ne sont, dans des mélanges avec de la cire d'abeille, apportés que par cette dernière (tabl. r).

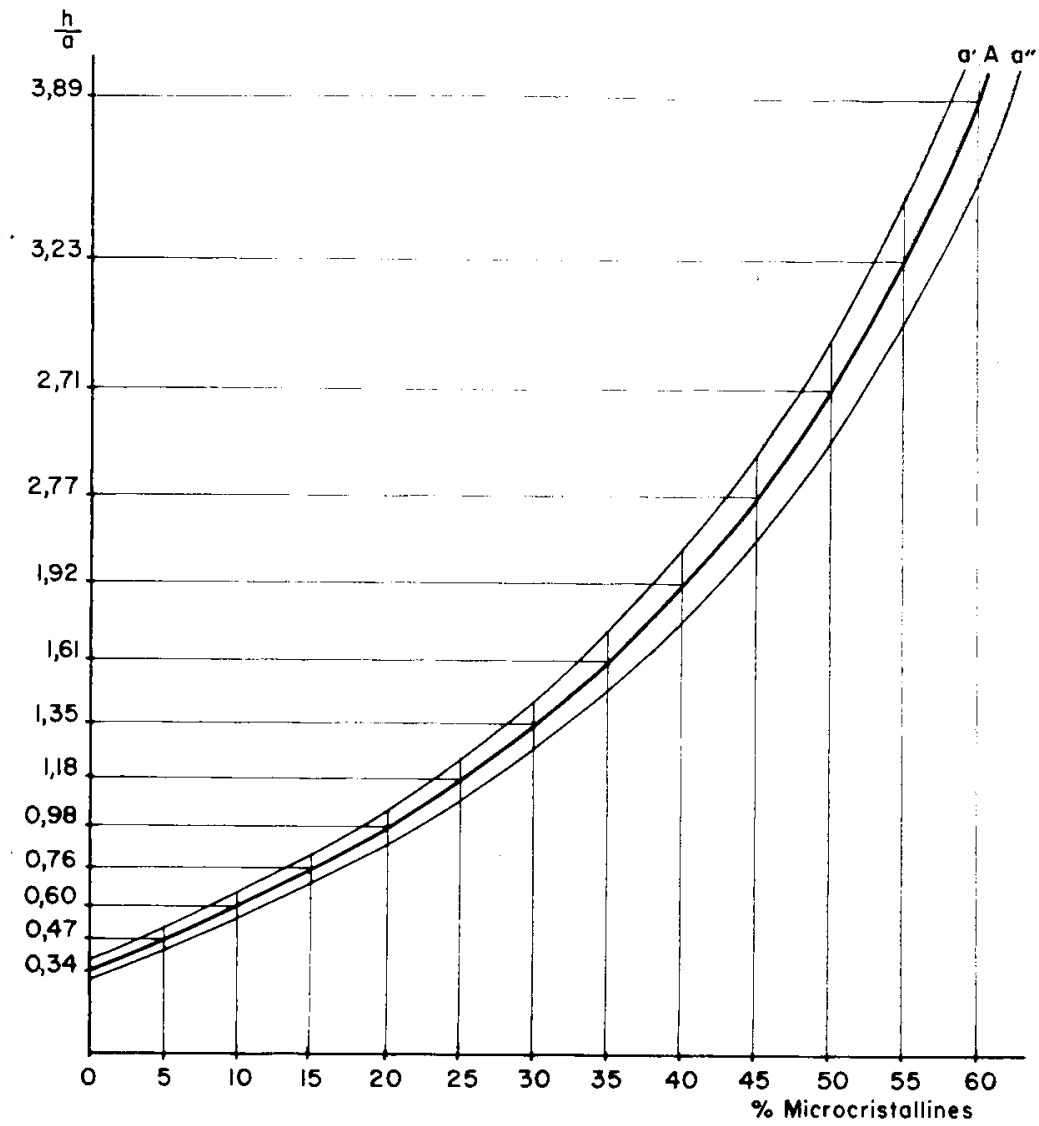

FIG. I. -- Variation du rapport ha en fonction de pourcentages croissants en microcristallines.

$h=$ teneur totale en hydrocarbures

$a=$ teneur totale en alcools gras.

Courbe A :

Teneur en hydrocarbures de la cire d'abeille $\ldots \ldots \ldots \ldots \ldots \ldots \ldots \ldots \ldots \ldots \ldots \ldots \ldots \ldots \ldots \ldots$

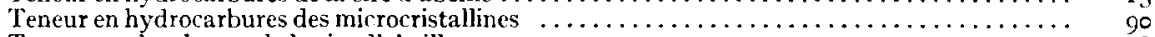

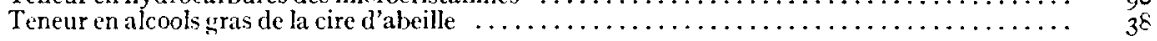

Courbe $a^{\prime}$ :

Teneur en hydrocarbures de la cire d'abeille $\ldots \ldots \ldots \ldots \ldots \ldots \ldots \ldots \ldots \ldots \ldots \ldots \ldots \ldots \ldots \ldots$

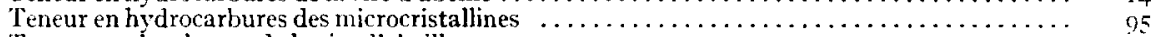

Teneur en alcools gras de la cire d'abeille . . . . . . . . . . . .

Courbe $a^{\prime \prime}$ :

Teneur en hydrocarbures de la cire d'abeille $\ldots \ldots \ldots \ldots \ldots \ldots \ldots \ldots \ldots \ldots \ldots \ldots \ldots \ldots \ldots$ I 2

Teneur en hydrocarbures des microcristallines $\ldots \ldots \ldots \ldots \ldots \ldots \ldots \ldots \ldots \ldots \ldots \ldots \ldots \ldots \ldots \ldots . \quad 8{ }^{2}$

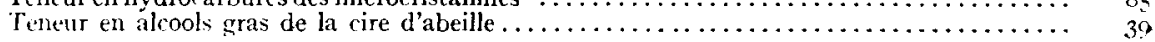


Le rapport $h$ a doit pouvoir donner une idée assez fidèle de la proportion de cires microcristallines dans un mélange. De plus, il a l'avantage de considérer deux familles de corps existant dans ces produits, ce qui donne plus de sécurité à l'emploi de la méthode que nous proposons.

\section{TABLEAU I}

\begin{tabular}{|c|c|c|}
\hline Cires & $\frac{\%}{\text { sanonifiable }}$ & Alcools \\
\hline Lamine blanche. . . . . . . . . . . . & 12.7 & 0 \\
\hline Laminée jaune .............. & 11,9 & 0 \\
\hline Micro $175 \ldots \ldots \ldots \ldots \ldots \ldots$ & 7,4 & 0 \\
\hline Vicro y $85 \ldots \ldots \ldots \ldots \ldots \ldots$ & 8,2 & 0 \\
\hline 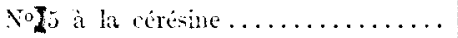 & 9,1 & traces \\
\hline , Warco raftinée............... & 12,9 & 0 \\
\hline i Warco 180 raftince $\ldots . . . \ldots \ldots$ & 11,9 & 0 \\
\hline Paraftines $\ldots \ldots \ldots \ldots \ldots \ldots \ldots$ & 1,5 & 0 \\
\hline
\end{tabular}

\section{2. - Préparation des échantillons.}

Ils sont lavés à l'eau chaude ( $\mathrm{\gamma}^{\circ}$ environ) et refroidis ; le gâteau cireux récupéré est đissous dans du benzène chaud. Ira solution obtenue est alors filtrée à chaud sur papier. Les particules non cireuses sont retenues. Les lavages à l'alcool éthylique ont été abandonnés à cause du passage en solution d'une partie de la fraction hydroxylée de la cire d'abeille.

\section{3. - Technique analytique.}

La technique analytique utilisée est celle d'Alexandre LEys (I9r3). L'échantillon de cire est dissous à chaud dans du benzène ( $\mathrm{log}$ dans $50 \mathrm{ml}$ ) et saponifié sous reflux par 25 cc de potasse alcoolique pendant 3 heures minimum. Le temps de saponification indiqué par LEIs ( $\mathrm{I} h$ ) est ainsi augmenté. La potasse alcoolique contient I $_{5}$ o g litre de potasse en pastille. Cette quantité élevée de potasse et le chauffage prolongé permettent une saponification complète des acides gras de la cire d'abeille.

La saponification est menée dans une ampoule à décantation chaude décrite par LEYS (voir fig. 2). Il s'agit d'un récipient de $45^{\circ}$ cc comportant iatéralement à la partie stıpérieure une ouverture rodée pouvant être adaptée à un bouchon de verre ou au réfrigérant à reflux et latéralement un robinet. Pendant la saponification, le robinet latéral est fermé. A la fin de la saponification, le réfrigérant est ôté et $50 \mathrm{ml}$ d'eau chaude sont ajoutés au mélange. Le réfrigérant adapté de nouveau, le chauffage est maintenu ro minutes.

Par la suite, le réfrigérant est définitivement ôté, le récipient bouché subit une rotation de $90^{\circ}$ de telle façon que le robinet se trouve à la partie inférieure, 1'appareil constituant alors une ampoule à décanter. Le liquide se sépare en deux couches ; une inférieure, correspondant à la solution savonneuse des acides qui est jetée une supérieure, benzénique, contenant l'insaponifiable qui est recueilli dans une capsule de porcelaine. 
Cet insaponifiable est dissous dans roo $\mathrm{ml}$ d'alcool amylique de la raçon suivante : la capsule étant disposée sur une platine chauffante, on verse les Ioo m1 d'alcool amylique par fractions, en ayant soin d'éliminer au fur et à mesure chaque fraction dans un erlenmeyer de 500 cc à large col. L'opération terminée, Ioo ml d'acide chlorhydrique pur sont ajoutés prudemment au mélange. Toutes ces opérations se font sur une platine chauffante. Cette dernière éteinte, le liquicle se refroidit doucement et les hydrocarbures surnagent. Il est alors facile de les séparer à l'aide d'une spatule de la bouillie cristalline d'alcools gras sous-jacente. I es alcools gras sont réchauffés avec un excès d'eau et ils surnagent à leur tour, constituant un gâteau à la surface d'une solution chlorhydrique. L,es produits obtenus doivent être lavés au benzène et chauffés jusqu'à disparition de toute odeur anylique. On obtient donc, en peu de temps et peu d'opérations, les hydrocarbures et les alcools gras.

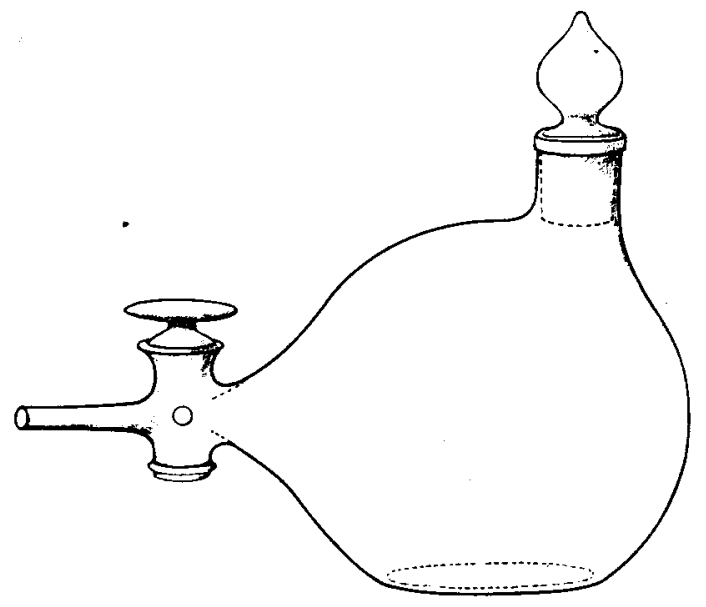

FIs. 2. - Appareil de Leys (IGI2)

La pesée de ces deux substances complexes ainsi obtenues permet d'établir le rapport $\frac{h}{a}$ ci-dessus défini.

4. - Établissement de la courbe.

Il nous a donc été possible de construire une courbe portant en abscisse la teneur en microcristallines et en ordonnées les différentes valeurs correspondantes de $\frac{h}{a}$.

Pour calculer ces différentes valeurs, il a été nécessaire de fixer des valeurs arbitraires probables.

La teneur moyenne en hydrocarbures de la cire d'abeille est très discutée. LEYs indiquait avec la méthode dont nous avons parlé, de I0,4à I3,03 p. cent (ce qui semble un peu faible), WARTH de I 3 à I 7 p. cent, WHITE et coll. de I 2,5 à I 4,5 p. cent C.ILLow 15 p. cent. Ein fait, les chiffres nettement supérieurs à I 5 p. cent nous semblent dus à des méthodes peu au point, à des accidents ou desinterférences dues aux colorants de la cire qui passent la plupart du temps avec les hỹdrocarbures (JAUBERT, I927 c; Bisson et coll., I940), colorants liés à la propolis et qui forment souvent une partie 
importante et variable de la cire d'abeille brute. Les lavages effectués au laboratoire semblent minimiser ces interférences. Nous avons trouvé des chiffres toujours voisins de I3,5 p. cent. Nous considérons que la valeur I3 p. cent est la plus probable.

Le taux en hydrocarbures des microcristallines est également variable.

Ainsi WAR'TH (I960) indique un taux d'huile de 5 à I 3 p. cent et WHITE de 9 à 20 p. cent (moyenne I5 p. cent), le premier grâce à des méthodes physiques, le second par chromatographie sur colonne. Au laboratoire, nous avons nous-même obtenu des résultats allant de $\mathrm{I} 2,7 \mathrm{p}$. cent (laminée blanche) à $7,4 \mathrm{p}$. cent (micro A 75). Il nous semble donc bien que ce taux d'huile oscille autour de ro p. cent (tabl. I).

Enfin, nous avons déjà vu que le taux en alcools gras de la cire d'abeille oscille autour de $3^{8} \mathrm{p}$. cent In définitive, nous avons construit une courbe en nous servant des chiffres suivants :

Taux en hydrocarbures de la cire d'abeille

I3 p. I00

Taux en alcools gras de la cire d'abeille

38 p. 100

Taux en hydrocarbures des microcristallines

go p. I00

I1 a été construit évidemment deux courbes limites tenant compte des variations possibles de ces chiffres.

Ainsi l'utilisation des trois courbes construites permet de connaitre une valeur movenne et deux valeurs limites sans autres calculs que celui du rapport $\frac{h}{a}$.

TABIEAU 2

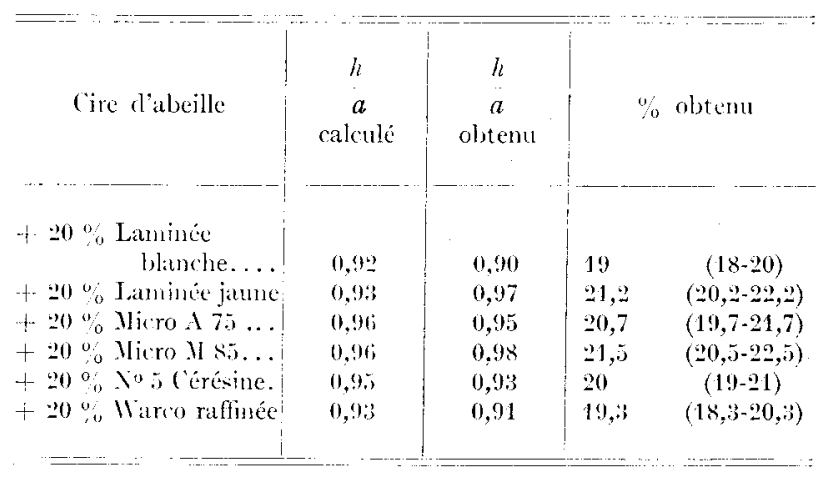

Cette courbe a été vérifiée expérimentalement en effectuant au laboratoire des mélanges de cire d'abeille et de cire microcrisalline de composition connue. I,e tableau 2 donne une idée des résultats obtenus. La cire d'abeille utilisée avait la composition suivante :

$$
\begin{aligned}
& h: 13,9 \text { p. cent } \\
& a: 38,4 \text { p. cent }
\end{aligned}
$$

\section{DISC'USSION}

Quelques objections peuvent être opposées à la technique proposée. İ̀n effet, le rapport $\frac{h}{a}$ déterminé expérimentalement n'est traduit en pourcentage de micro- 
cristallines que grâce à des considérations théoriques. Mais cela ne peut pas ètre évité. Les produits étudiés ont une composition variable et des chiffres de base hautement probables sont nécessaires. Par la suite, la considération des variations possibles est indispensable ; c'est pour cela qu'à la courbe moyenne nous ajoutons deux courbes limites permettant de prévoir un intervalle de confiance raisonnable. Cet intervalle n'excède jamais 3 à $4 \mathrm{p}$. cent ce qui, pour un dosage de contrôle, est largement suffisant. Lorsque les ordonnées croissent très rapidement pour des teneurs en microcristallines élevées, l'imprécision relative augmente. Par contre, nous isolons directement par cette méthode deux familles de corps, ce qui permet d'obtenir des résultats plus nets que par dosage des fonctions chimiques, qui sur des corps aussi complexes, peu réactifs et peu connus, n'ont que peu de signification (Bissor et call., I940).

Enfin, en cas de légères pertes au cours des opérations analytiques, en particulier au moment de l'extraction de l'insaponifiable, les résultats sont peu modifiés ; en effet, seul le rapport entre hydrocarbures et alcools gras entre en jeu.

L,a méthode ainsi exposée semble donner une solution au problème du contrôle des produits vendus sous l'appelation " cire d'abeille " et susceptibles d'être fraudés par des cires microcristallines. Un dosage peut facilement être réalisé en une demijournée. La précision de 3 à 4 p. cent semble, dans l'état actuel des choses, suffisante pour le contrôle des produits actuellement commercialisés.

Il est à noter que la méthode proposée n'est valable que pour un mélange de cire d'abeille et de cire microcristalline. En effet, si une troisième substance apporte soit des alcools gras, soit des hydrocarbures, il devient impossible de tracer la courbe. Ie toute façon, il est toujours indiqué de considérer en plus du rapport la somme $h+a$ afin de vérifier la vraissemblance de la solution proposée.

Enfin, notons que l'addition de cire de Carnauba (très riche en alcool gras) entraînerait une diminution du rapport $\frac{h}{a}$ de la cire d'abeille. Mais ce cas n'a pas été étudié ici.

Reçu pour publication en juin 1964 .

\section{SUMMARY}

DETERMINATION OF THE CONTENT OF MICROCRYSTAILINE WAX

IN MIXTLRES OF BEESWAX AND MCROCRYSTALIJNE WAXES

A comparative study of the chemical composition of beeswax and microcrystalline waxes shows that fatty alcohols are present only in the former. A more detailed chemical study of microcrystalline wases leads to the conclusion that the average content of hydrocarbons in these materials is abuut 90 p. 10o. The ratio:

$$
\frac{\text { content of hydrocarbons }}{\text { content of alcohols }}=\frac{h}{a}
$$

of a mixture can therefore give an idea of the proportions of microcrystalline waxes and beeswax.

These contents are obtained by the method of A. LEYs (I9I3) : the fatty alcohols and the hydrocarbons in the unsaponifiable matter are separated by the amyl alcohol/hydrochloric acid mixture.

A theoretical curve (and two limiting curves) can be plotted to show the variations of the ratio defined above as a function of the amount of microcrystalline waxes added to becswax. This curve has been verified by experiment. 


\section{RÉFÉRENCES BIBLIOGRAPHIQUES}

Bisson C. S., VANsent G. H., Dye W. 13., I940. Tnvestigations on the physical and chemical properties of Beeswax. U.S. Depart. Agric. Tech. Bull., $7 \mathrm{I} 6$.

Caillas A., 1945. Les produits de la ruche. Orléans.

Callow R.K., I963. Chemical and Biochemical problems of Beeswax. Bee Iforld, 44 (3), 95-101.

Chauvin M., 1955. Fabrication des paraffines, pétrolatum et cires à partir du pétrole brut aramco. fe Congrès mondial du pétrole. Actes et documents. VI. Utilisation des produits du pétrole. 67-73. Rome.

Damor G., 1924. Contribution à l'étude chimique de la cire d'abeille. Thèse PT'F, Paris, i94.5.

Ellis C., 1945. The chemistry of Petroleum derivatives. Reinhold, New Iork.

Fuchs W., Joxg A. de, 1954. Chromatographische Zerlegung. Fette Seiten, 56 (4), 2 I 8-22o.

Gascard, 1920. Recherches sur les termes élevés de la série grasse saturée. Thèse, Faculté des Sciences, Paris.

Gruse W. A., Stevens O. R., 1960. Chemical technology of Petrolem. IIc. Graw-Hill, New York.

JAUBERT Georges F., I927. Sur l'origine de la coloration de la cire d'Abeille et la composition de la propolis. C. R. Acad. Sci. Paris, 184, г I34-1 I 36 .

KANE, 1960. Petroleum Waxes quality requirements past, present and future. Tational petroleum symposium, 57 th annual meeting. Cleveland, april 20th 1950 . National Petroleum Association.

Leys A., 191 2. Cire d'Abeille et de Carnauba. J. Pharm. chim., 5, 5i-588.

LEys A., r9ı3. Cire d'Abeille et de Carnauba. Méthode d'analyse. Dosages des hydrocarbures étrangers. Ann. Chim. anal., 17, 334-3+2.

Ludecke C., IvanovszkyL L., 1958. Taschenbuch fïr die Wachs Industrie. Stuttgart.

Mazee W. M., 1949. On the properties of paraffin wax in the solid state. $J$. Iust. Petroleum, 35, $97^{-102 .}$

PARker H., R., 1960. Saponification cloud test for beeswax. Irug. Stand. 28 (1), 26-20.

Root H. H., I95I. Beeswax : its properties, testing, production and applications. Brooklyn, New York.

Spengler G., Weber, tg62. Bestimmung das Bienen Wachsgehalt in Wachs Komposition aus gesant paraffin, verseifungs. Ester- und-Säure-sahl. Fette Seifen, 62 (1o).

Turner W. R., Brown D., S. Harisox D. V., i955. Properties of Parafin waxes. Ind. eng. Chem. 47, I 2 I9.

Wartu A. H., 1960. The chemistry and Technology of waxes. 940 p; Reinhold, New York.

White J. W., Reader M. F., Rietiof M. T., ig6o. Chromatographic Determination of Hydrocarbons in Beeswax. J.ass. Off. Agric. Chem., 43.

White J. W., RiethoF M. L., Kusinir I., 1060. Estimation of nicrocrystalline wax in Beeswax. J. ass. Off. Agric. Chem., 43.

White J. W., Kushnir I., Analysis of mixtures of Jeeswax and l'etroleum waxes. Amer. Bee f., 101 (t), I 8-2I. 